\title{
Unexpected behaviour of microbubbles immediately at the aerator exit
}

\author{
V. Tesar̆ \\ Institute of Thermomechanics ASCR v.v.i., Prague, Czech Republic
}

\begin{abstract}
Microbubbles (of diameter less than $1 \mathrm{~mm}$ ) are desirable for many engineering applications. Until quite recently, generating them efficiently was a problem. A solution was found in excitation of gas supply by inexpensive and reliable nomoving-part fluidic oscillators. Strangely, generated microbubbles are larger than expected. The author studied their formation using a high-speed camera with a long distance macro-lens. The images revealed the cause being a conjunction of bubbles, mostly taking place within the very proximity of the aerator exit. Small bubbles move slowly and do not vacate fast enough from the position in which they were generated. They thus get into contact with the next generated bubble. Another cause of slow departure is the increased drag caused by shape oscillation driven by energy released in the conjunction. The conjunctions may also occur due to lateral bubble motion if the Reynolds number exceeds the vortex shedding threshold.
\end{abstract}

Keywords: bubbles, microbubbles, bubble conjunctions.

\section{Introduction}

Dispersion of a gas in liquid in the form of bubbles is of paramount importance for a large number of applications in chemical and process engineering, biotechnology, food sciences and medicine. Supplying air bubbles through an aerator in waste water processing, or $\mathrm{CO}_{2}$ bubbles for growing algae are two most common examples. Many of these processes could be more efficient if the size of the bubbles was smaller. However, the total surface energy of gas volume distributed into many smaller bubbles is inevitably larger and this means more energy must be spent on their generation. This is particularly true for microbubbles, of equivalent diameter less than $1 \mathrm{~mm}$. Typical microbubble 
generation mechanisms used so far (such as ultrasound, saturation nucleation, or Venturi effect) are also rather inefficient. As a result, microbubbles while a topic of discussion in engineering circles for a decade, have been seldom practically used. The situation has changed mainly due to the research work carried out by Prof. Zimmerman and his team at the University of Sheffield [1, 2]. The key to the new situation is the oscillation in the gas supply into the aerator. Particularly advantageous for this purpose is the use of no-moving-part fluidic oscillators. The absence of mechanical (moved or deformed) components results in practically unlimited life, reliability, low price and other advantages. No electric current is needed (an advantage in the harsh environment in, e.g., waste water processing). The hopes associated with this new idea have even led to the promise of generating the so far somewhat enigmatic and elusive nanobubbles $[3,4]$. In some respects, the microbubbles generated by fluidics can even exhibit behaviour qualitatively different from that of coarse bubbles - an example is the direct contact evaporation where it was demonstrated [5] that microbubbles make possible delivering heat into the requested vaporisation without wasting energy on heat transfer into liquid.

In the struggle for obtaining microbubbles it has been somewhat disconcerting that the diameters of the bubbles produced by fluidic excitation of air flow passing through the aerator exits were significantly larger than the exit size. Even with aerator orifices of equivalent diameter $\sim 0.1 \mathrm{~mm}$ or less the typical mean size of generated microbubbles is rarely less than $\sim 0.5 \mathrm{~mm}$.

\section{Experiment}

To investigate the reason for the larger than expected size of microbubbles, the author studied their formation and subsequent behaviour at and near the aerator exit by a high-speed camera using a long-distance macro-lens that made possible taking images from a distance $\sim 100 \mathrm{~mm}$. The sub-millimetre scene could be thus recorded from outside, through the glass wall of the vessel. The recording speed was either 1000 or 4000 frames per second (the limiting factor was available light that had to be focused on the very small scene). This led to the discovery of unexpected very fast effects. The bubble gas was air, generated in water at $130 \mathrm{~mm}$ depth below surface by blowing through an aerator in the form of a glass frit disk of $5 \mathrm{~mm}$ thickness. The frit-sintered glass dust had narrow passages of equivalent diameters approximately $0.1-0.2 \mathrm{~mm}$. The aerator top surface, where the microbubbles were generated, was positioned horizontally, with air supplied from below.

The lighting of the scene was applied from above, by white LEDs shining on the white top surface of the frit. This resulted in the white reflection at the bottom part of the photographed bubbles while their top half was dark. At the small vertical distances of interest above the frit, the background colours were various degrees of gray (scattered light reflected from the frit). This made possible quite precise detection of the bubble centres in the camera images in the subsequent manual image processing. The size of the bubbles and the vertical distances of their centres from the aerator exit were measured in individual 
frames. With precise time measurement available in the camera records, the bubble motion was evaluated from height/time bubble trajectories.

\section{Ascent of bubbles in a chain}

Bubbles initially grow attached to the aerator exit and if positions of their centres are identified in the images and plotted, their height above the aerator increases, although this of course is not a motion of the bubble as a whole. The corresponding initial part of the height/time trajectory is usually distinctly different (as may be seen in Figs. 2 and 5) from the subsequent parts of the trajectory after the bubble separates and moves upwards.

The velocity of the ascent depends very much on the bubble size: small bubbles move very slowly. At any rate, the velocity is very low at the beginning, due to the need to overcome the inertia of water that surrounds the bubble. This water has to be accelerated and as a result the bubble only gradually reaches its terminal velocity at which the Archimedean lift is in balance with the drag force. As a matter of fact, the drag force slightly varies with bubble velocity so that the concept of terminal velocity is not strictly and precisely applicable - but it may provide a useful approximate reference.

Typical for large bubbles, of diameters larger than $1 \mathrm{~mm}$, are ascent velocities so high that the bubble gets far away from the aerator before the subsequent bubble is formed. Individual bubbles from the same aerator exit do not influence one another. On the other hand, when a microbubble separates from the aerator exit, its slowly ascending predecessor is still within the aerator proximity. Thus microbubbles typically rise to the surface in lines (sometimes called chains or strings), as shown in the video still presented in Fig. 1(a). Dynamics of microbubble ascent may be quite strongly influenced by the wake of its predecessor - depending, obviously, on the pitch $l$, the distance between each two bubbles in the chain. It has to be said that although it is not very apparent, a closer study of Fig. 1(a) reveals that with the increase of velocity approaching the "terminal" velocity the pitch distances $l$ increase from the bottom to the top of the image (evaluation of Fig. 1(a), for example, shows the pitch $l$ in this case having increased from initial $0.255 \mathrm{~mm}$ to $0.3 \mathrm{~mm}$ at the top of the image). Of course, the absolute magnitude of the pitch $l$ is not so much important as is its relative value $\lambda$, related to the bubble diameter $d$. Usually, the relative pitch $\lambda$ is evaluated for the more or less stabilised conditions at the largest available vertical distance from the aerator. In the case presented in Fig. 1(a) the bubble diameter is $d=0.155 \mathrm{~mm}$ so that the characteristic relative pitch $\lambda$ is 1.9 .

Theoretical terminal velocity $\mathrm{w}[\mathrm{m} / \mathrm{s}]$ may be evaluated for a solitary solid body moving in the liquid (water) at a low Reynolds number $(\operatorname{Re}<1)$, using the Stokes law [6] for the drag coefficient

$$
\mathrm{C}_{\mathrm{D}}=24 / \mathrm{Re}
$$

- or the Massey's empirical relation [7] for $\mathrm{Re}<100$

$$
\mathrm{c}_{\mathrm{D}}=(24 / \mathrm{Re}) *(1+93 * \operatorname{Re} / 16)^{0.5}
$$


In light of what will be discussed in Sect. 6 of this paper, it should be noted that the terminal-velocity Reynolds number of the bubbles shown in Fig. 1(a) is $\mathrm{Re}=5.7$. The dependence of the terminal velocity $\mathrm{w}$ on the bubble diameter $\mathrm{d}$ evaluated on the basis of eqs. (1) and (2) is presented as the heavy line in Fig. 1(b).

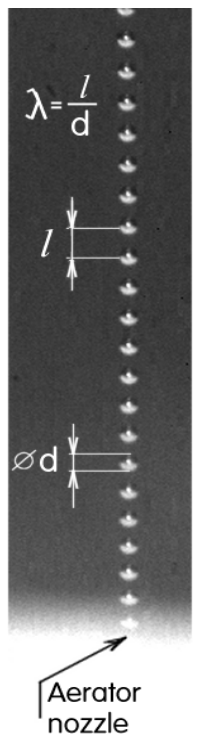

(a)

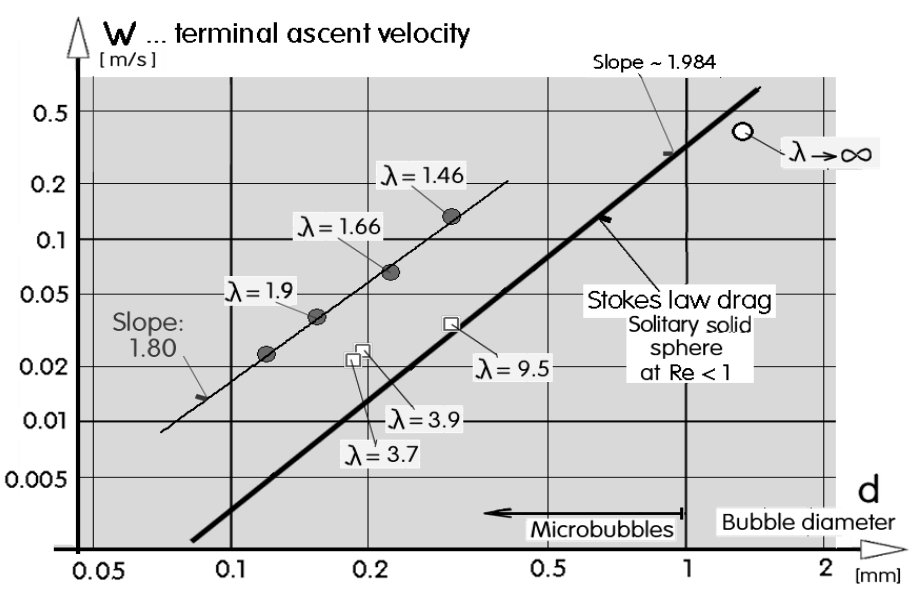

(b)

Figure 1: (a) Photograph of microbubbles rising in a line to the surface. The ascent velocity is strongly influenced by the relative pitch magnitude $\lambda$. (b) Dependence of measured terminal velocity of the ascent on the bubble diameter. The heavy line as a power-law fit is for reference, evaluated by equality of Stokes drag and Archimedes lift forces. The thin lines connect the present author's experimental data for ascent in small-pitch bubble chains.

It is a useful reference for approximating the bubble ascent behaviour. In particular, it should be noted (Fig. 1(b)) how rapid is the decrease of the ascent velocity with decreasing bubble size. Actual bubbles, of course move differently - some earlier information on their velocities is in refs. [8-11]. In general, experimental data from literature show the ascent velocities of solitary bubbles to be slightly lower than the prediction following from the eqs (1) and (2) for a solid body. For example, in a diagram arranged as similar to Fig. 1(b), LigerBelair et al. [8] presented measurement results obtained with the small bubbles in champagne. The data are rather scattered; nevertheless they may be described as occupying a band of velocities 0.6 to 0.8 -times slower than the solitary solid sphere predictions. 
Also the present author's evaluations from camera images for the case of the coarse bubble (note that it is not a microbubble) in the upper right-hand corner of the diagram in Fig. 1(b) agrees (by being below the line fitted to the Stokes law) with these conclusions. In this large bubble case, the relative pitch $\lambda$ in Fig. 1(b) is labelled as infinite. These bubbles move so fast that they are not influenced by the wake of its predecessor bubble.

In other, microbubble cases of the author's camera records, it is obvious that the motion in the string makes the upward motion of bubbles easier. Their velocity was evaluated from the slope of the height/time trajectories, which show that the data may be roughly grouped into three sets, depending on the magnitude of the relative pitch $\lambda$. There is in Fig. 1(b) a single case of mutually very distant bubbles, $\lambda=9.5$. This relative distance is longer than the downstream reach of the wakes of predecessor bubbles. As a result, the velocity is only a little higher than the prediction for solitary solid body. An obviously more pronounced effect was found for the two cases with $\lambda=3.7$ and $\lambda=3.9$. Very probably, the wakes fill there the spaces between consecutive bubbles so that the whole chain can reach a higher terminal velocity.

Finally, there are in Fig. 1(b) the four cases of very short distances between the bubbles. Even though the relative pitch values $\lambda$ were not strictly equal, their mechanisms of the liquid in the short spaces moving with the bubbles, facilitating the upward motion, must be quite similar - to the degree of enabling a common power-law line fit through all four data points (the thin line with slope 1.80). Typically, the velocities are 4.5-times higher than the Stokes-law line.

\section{Conjunctions}

The rapid decrease of the ascent velocity with decreasing bubble size means the speed with which a microbubble vacates the space at the aerator channel exit where it was generated - is typically quite slow. So slow in fact that the bubble may interfere with its follower bubble. They conjugate - in a process that is apparently very fast (so that its details were never captured even by the used very high-speed camera). The ascending microbubble chain thus consists of doublesize bubbles. The effect is presented in Fig. 2 both by selected four photographs and by the height/time trajectories. It has apparently never been so far mentioned in literature where it seems the opinion prevailed that bubbles simply cannot be generated comparable in size with the aerator exit. The increase in bubble size caused by the conjunction is not immediately apparent, because bubble volume is proportional to the third power of the diameter (the $V \sim d^{3}$ law). Thus doubling the volume increases the bubble diameter only 1.26 times, an increase that may be not conspicuous. What should be particularly emphasised is the small height above the aerator exit at which the conjunction process takes place. The process as shown in Fig. 2 happens at a height of a fraction of a millimetre. Usual measurements of bubble size are never made by unsuspecting researchers at these heights, but always at heights equal to several millimetres. Standard methods actually do not allow size measurements at sub-millimetre heights. Commonly used size measurement methods are optical - using the laser light 
scattering on the bubbles - and in the presence of the wall the results deteriorate (e.g. by diffraction or reflections). No wonder the results indicate the large bubble size. Another aspect is the speed of the conjunction effect. It cannot be observed by the eye and was discovered thanks to the unusual use of the highspeed camera. The frequency of the consecutive release of the small primary bubbles from the aerator exit, $150 \mathrm{~Hz}$ in Fig. 2 (or even higher e.g. $542 \mathrm{~Hz}$ in Fig. 6), obviously cannot be observed by the eye.

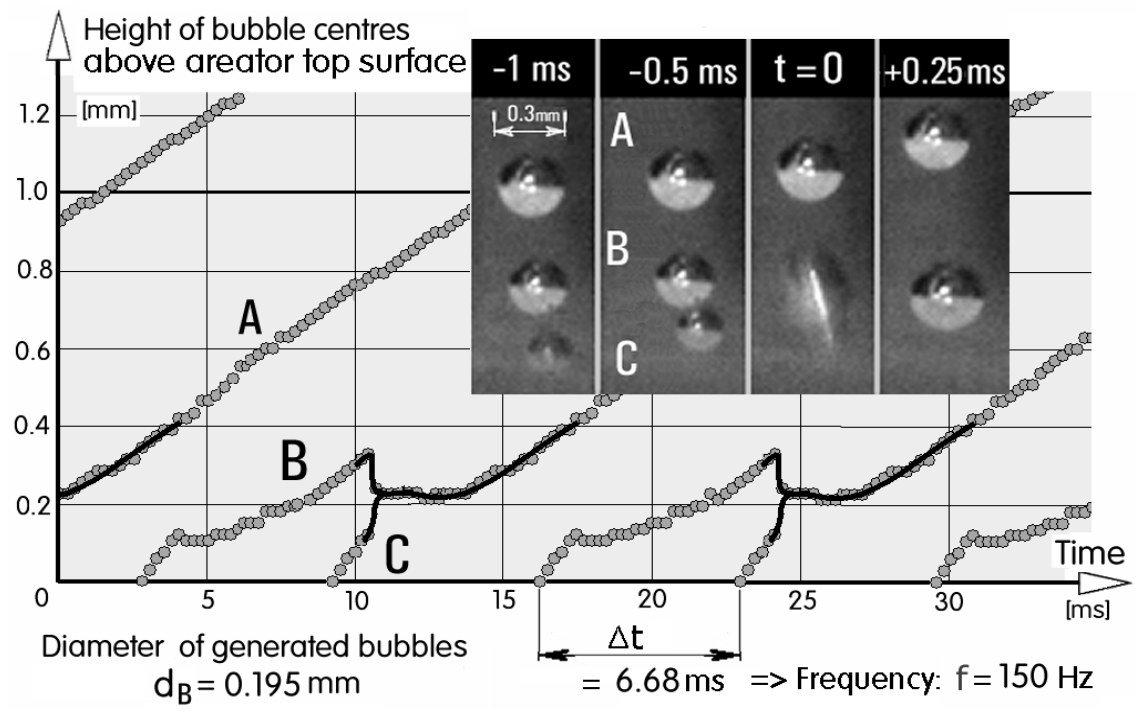

Figure 2: $\quad$ Trajectories height/time - measured manually in individual camera images - evaluated for the quite typical case where two bubbles initially generated as separate entities undergo a conjunction. The inset shows photographs of three bubbles, marked A, B, and C. All larger bubbles $A$ consist of two merged bubbles $B$ and $C$.

The doubling of bubble volume, presented in Fig. 2, is by far not the worst among the hindrances encountered in producing the desired small microbubbles. Quite often the resultant bubble contains, as a result of several consecutive conjunctions, air from a number of primary bubbles. A photographic document example is presented in Fig. 3(a). The video clip revealed the bubble $A$ is a result of three consecutive conjunctions of the primary bubbles $C$ (so that the volume of $A$ corresponds to four volumes of primary bubbles $C$ ). Note the three missing positions in the bubble chain above $A$. Of course, because of the $V \sim d^{3}$ law, the four-times increase in the volume increases the radius of $\mathrm{A}$ less conspicuously to only 1.58 times the radius of $\mathrm{C}$.

In Fig. 3(b) the height/time trajectories document an observed (and recorded) growth of a bubble by conjunctions so that it is finally formed by six coalescing primary small bubbles. As in the previous example, the processes take place at 
sub-millimetre heights above the aerator exit and are so fast that they inevitably escaped attention.

Some unexplained questionable facts remain. Sometimes the conjunction is avoided without any apparent reason. Thus in Fig. 3(a) there are four primary bubbles at the top of the image that escaped unaffected prior to the conjunction of the four later ones that coalesced into the bubble $\mathrm{C}$. In a similar manner, in the example presented in Fig. 3(b) the trajectories show only triple conjunction immediately before the six-fold conjunction - and mere doubling thereafter.

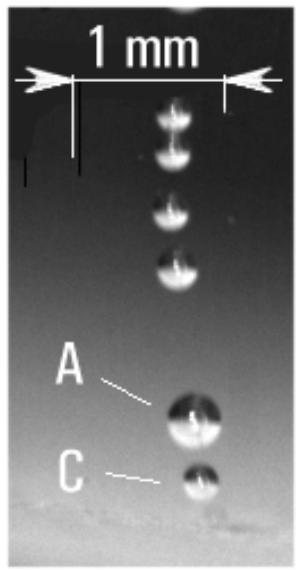

(a)

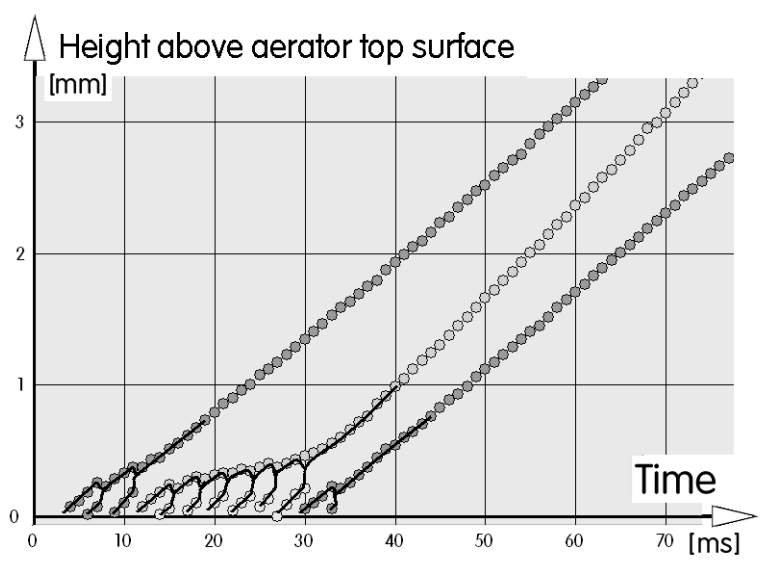

(b)

Figure 3: (a) Multiple conjunctions near the exit: the bubble $A$ in this photograph is a result of sequential conjunction of four bubbles $C$ (note the space between $A$ and its predecessor above due to missing three primary bubbles that coalesced with the gradually growing $A$ ).

(b) Height/time trajectories of six-fold multiple conjunction.

\section{Drag increased by oscillation}

After many cases of conjunction (though not all of them) the formation of the resultant large bubble is followed by its rather vigorous oscillation. An example is shown in Fig. 4. Explanation is based on considering the energy aspect of the process. If two bubbles merge, the surface of the resultant bubble is smaller than the sum of the surfaces of the original pair. This means surface energy - which is proportional to the surface area - decreases in the conjunction process. This is the reason why bubbles merge so eagerly: the system strives to get to the lowest accessible energetic level. What drives the oscillation is the energy released in the conjunction process. Oscillation is typically visible in the high-speed camera records where it may be recognisable for a considerable time until it is finally dissipated by viscous friction in liquid $[12,13]$.

Fig. 4 demonstrates that the oscillation in question is characterised by variations in the bubble shape - in other words departures from sphericity. This is not surprising because the starting configuration of the two bubbles in contact 


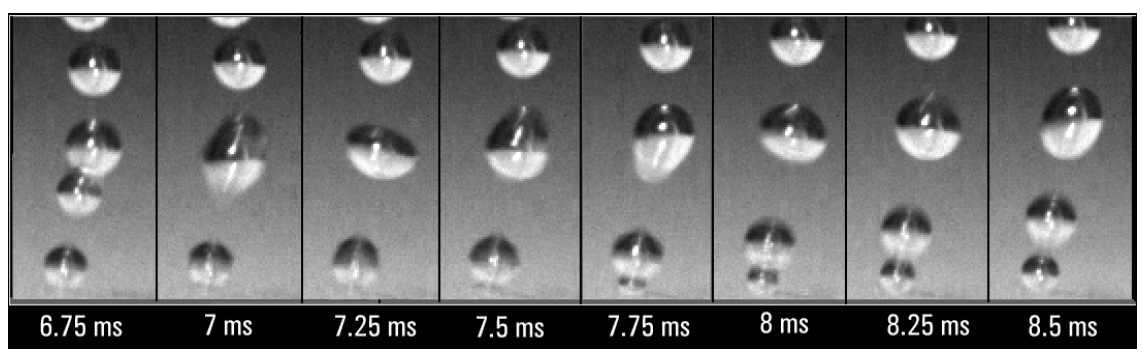

Figure 4: An example of conjunction of the two bubbles (seen in the leftmost image) followed by oscillation of the resultant large bubble driven by released surface energy.

is asymmetric (aspherical). Shape oscillation is described by strongly non-linear differential equations of radial bubble dynamics - presented, for example in ref. [14] - admitting no analytic solution in terms of simply defined functions. Because of the difficult character, studies existing in literature mostly concentrate on the simplified problem of stability as a condition for the oscillatory motions $[14,15]$ rather than the subsequent oscillation of finite amplitudes. The basic spherical bubble shape imposes a periodic character of the boundary conditions in the form of distinct oscillation modes for standing waves. Obvious in Fig. 4 is the trend to select the lowest of the accessible modes, the one characterised by only two excrescences located on the opposite sides of the bubble. The surface of an aspherical shape is, of course, larger than that of a spherical bubble - so that the surface tension, tending to minimise the surface area, provides the directive force returning back the excursions from sphericity.

Much more attention in literature on oscillation of bubbles was directed to understanding the bubbles oscillating in their volume while retaining the spherical shape. This has been studied since the time of Lord Rayleigh in 1917 [16] whose basic equation is still cited in current publications. Because of the spherical shape, the bubble volume dynamics is fully described by temporal dependence of bubble diameter (or radius in [16]). As a result, Rayleigh's equation is an ordinary differential equation, much easier to solve. It assumes, however, very high changes in gas pressure inside the bubble - while in the present case, with the deformed shapes, the pressure may remain essentially constant. Theory of the shape oscillations is far less developed than that of the volumetric case (partly because of the simpler character and partly due to the need to understand cavitation). Characteristically, the extensive reference [17] summarising the results of all bubble oscillation research devotes only a very small part of its contents (roughly one page out of 88) to the shape oscillation.

The fact of importance in the context of the present paper is the higher hydrodynamic drag of the rising oscillating bubble. This is clearly demonstrated, for example, in Fig. 5. The first generated primary bubble rises at $0.267 \mathrm{~m} / \mathrm{s}$. It may be rightfully expected (on the strength of the general dependence of ascent velocity on the bubble size in Fig. 1(b)) that the larger bubble produced by the 


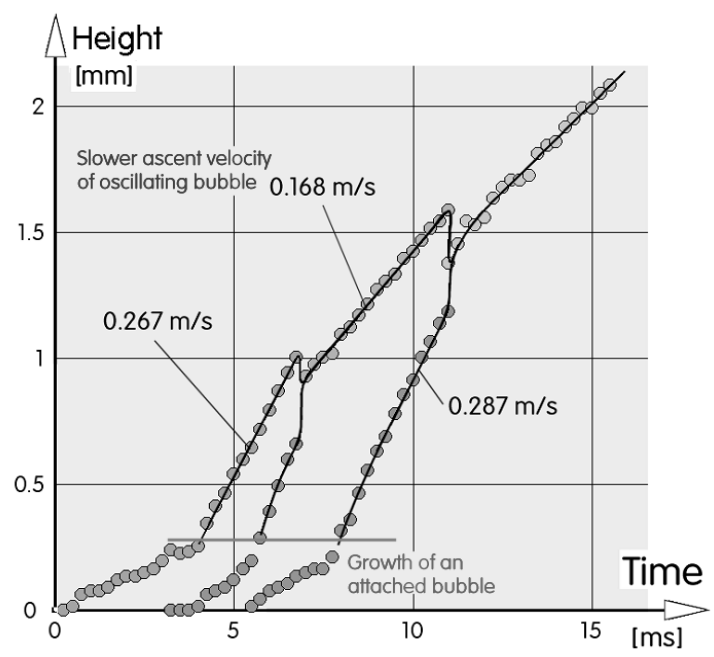

Figure 5: A general rule demonstrated in Fig. 1(b) is that larger bubbles ascend faster. There is, however, an exception if the larger bubble created by conjunction oscillates (Fig. 4). It may then rise so slowly that it can - as this height/time trajectories demonstrate - coalesce with a bubble generated much later.

conjunction should ascend faster. Instead, at $0.167 \mathrm{~m} / \mathrm{s}$ its velocity is substantially slower - by an amount so large that it cannot be explained by the increased pitch in the bubble chain. This decrease in the ascent velocity may be so significant that the height/time trajectories in Fig. 5 show its subsequent conjunction with yet another, later generated primary bubble. Somewhat surprisingly, this conclusion of the increased drag due to oscillation is far from obvious. Experiments by Wu and Gharib [18] with rising bubbles of equivalent diameter range $\mathrm{d}=1-2 \mathrm{~mm}$ in still water have been interpreted as an oscillating bubble being twice as fast as the unperturbed bubble of the same volume. These results were considered surprising by other authors, and refuted, for example by [19] - who, however, in their detailed force balance came with another surprising idea: a claim that the velocity decrease is due to added mass rather than the hydrodynamic drag.

\section{Lateral motions}

Only microbubbles ascending at very low Reynolds number of the upwards motion (typically at $\mathrm{Re}<35$ ) do so in a line or chain, a typical example of which is shown in Fig. 1(a). At these low Reynolds numbers the wakes trailing behind the bubble remain attached. Once this limit value is reached or surpassed, the wakes are shed. The shedding is asymmetric: a part of the wake separates first before it is joined by the other part. The flow past the bubble obtains a sideways impulse. The bubble then finds itself away from the vertical line 
(bubble chain) in which the ascent is made easier by the interaction with the wake of the predecessor bubble. It is also quite probable that the bubble exposing its side to outer slower liquid undergoes a rotation pushing it even more away from the chain. At any rate, the action on the displaced bubble comes rapidly. And almost instantly it pushes it towards the follower bubble, often with a subsequent junction process. Before the importance of this displacement was established, it seemed strange why sometimes, for example in Fig. 6, a conjunction is observed between a pair of microbubbles that up to the conjunction exhibit a practically equal ascent velocity.

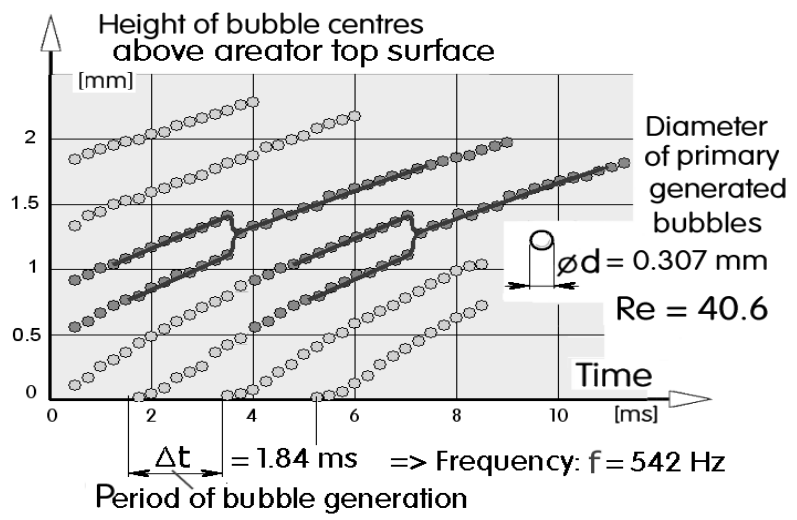

Figure 6: Seemingly strange case of junction, rather high above the aerator exit, of two bubbles having practically equal ascent velocities.

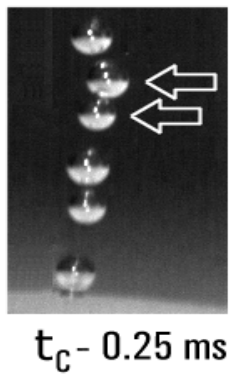

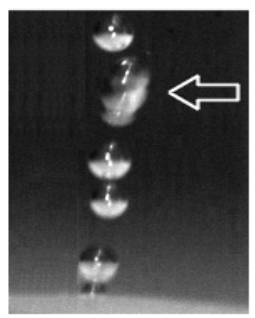

time: $t_{C} \quad t_{C}+0.25 m s$

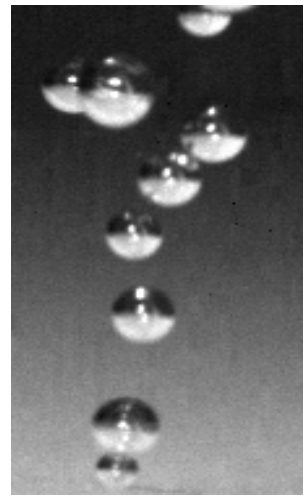

Figure 7: (a) Photographic record of the coalescence from Fig. 6. An explanation for the two bubbles meeting (and coalescing into a single large one) is in the transversal motion caused at $\mathrm{Re} \geq 40$ by asymmetric shedding of wake vortices. (b) An example of bubble string breakup caused by lateral motions at Re high enough for vortex shedding. Once a bubble leaves the vertical line, its ascent velocity decreases - cf. Fig. 1(b). 


\section{Conclusions}

The paper analyses accumulated video clips material obtained with the highspeed camera in an attempt to explain the fact that typical microbubbles are significantly larger than the size of the aerator exit at which the microbubbles are generated. The images and their manual processing into height-time trajectories clearly reveal that the reason is bubble conjunction, mostly taking place within the very proximity of the aerator exit. The basic reason why bubbles do merge is their high surface energy, which is released once the bubbles come into mutual contact. This getting into contact is made not accidentally, but in a quite systematic manner (sometimes by multiple conjunctions, one immediately after another) because ascent velocity of microbubbles is generally very slow and does not suffice for the bubble vacating its position near the aerator exit before the follower bubble grows to the separation size. Bubbles may get into contact, with subsequent conjunction, also due to their oscillatory motion caused by the surface energy released a moment ago. An important fact contradicting the coalescence is organisation of the bubbles into a chain or string in which particularly if the relative pitch between them in the chain is small - their drag is decreased by an interaction of the predecessor bubble wake. A bubble may get outside the chain by the asymmetry of the wake shedding if Reynolds number of bubble motion exceeds the vortex shedding threshold, at about $\operatorname{Re}=30-40$.

\section{Acknowledgements}

The author is grateful to Dr Jiř́ Šsonský for making available the very high-speed camera and the software for image processing. Experimental investigations were supported by grant Nr. 13-23046S received from GAČR - the Czech Science Foundation.

\section{References}

[1] Zimmerman W., et al. Microbubble generation, Recent Patents in Engineering. 2008; Vol. 2: p. 1

[2] Zimmerman W., et al. On the design and simulation of an airlift loop bioreactor with microbubble generation by fluidic oscillation. Food Bioprod. Process. 2009; Vol. 87: p. 215

[3] Zimmerman W. et al. Towards energy efficient nanobubble generation with fluidic oscillation. Current Opinion in Colloid \& Interface Science, 2011; Vol. 16; p. 350

[4] Wu C., Nesset K., Masliyah J., Xu Z. Generation and characterization of submicron size bubbles. Advances in Colloid \& Interface Science, 2012; Vol. 179; p. 123

[5] Zimmerman W. et al. Evaporation dynamics of microbubbles, in Print, Chemical Engineering Science 
[6] Stokes G. G., On the effect of the internal friction of fluids on the motion of pendulums, Transactions of the Cambridge Philosophical Society, 1854, Vol. X, p. 8

[7] Massey B. S., Mechanics of Fluids, 6th Ed., Van Nostrand Reinhold (International), page 172, 1989

[8] Liger-Belair G., et al. On the velocity of expanding spherical gas bubbles rising in line in supersaturated hydroalcoholic solutions: Application to bubble trains in carbonated beverages, Langmuir, 2000; Vol. 16, p. 1889

[9] Celata G.P., et al. Measurements of rising velocity of a small bubble in stagnant fluid in one- and two-component systems, Experimental Thermal and Fluid Science, 2007; Vol. 31, p. 609

[10] Di Marco P., et al., Experimental study of rising velocity of nitrogen bubbles in FC-72, Internat. Journ. of Thermal Sciences, 2003; Vol. 42, p. 435

[11] Hepworth N.J., et al., Characterizing gas bubble dispersions in beer, Food and Bioproducts Processing, 2001; Vol. 79, p. 13

[12] Roberts P.H., Wu C.C. The decay of bubble oscillations, Physics of Fluids. 1998; Vol. 10, p. 3227

[13] Aganin A.A., et al., Effect of fluid viscosity on the decay of small distortions of the gas bubble from a spherical shape, Journal of Applied Mechanics and Technical Physics 2006; Vol. 47, p. 175

[14] Strube H.W., Numerische Untersuchungen zur Stabilität nichtsphärisch schwingender Blasen, Acustica 1971, Vol. 25, S. Hirzel Verlag, Stuttgart

[15] Hao Y., Prosperetti A., The effect of viscosity on the spherical stability of oscillating gas bubble, Physics of Fluids 1999; Vol. 11, p. 1309

[16] Lord Rayleigh, On the pressure developed in a liquid during the collapse of spherical cavity, Philosophical Magazine Ser. 6, 1917; Vol. 34, p. 94

[17] Lauterborn W., Kurz T., Physics of bubble oscillations, Reports on Progress in Physics, 2010; Vol. 73, p. 106501-106589

[18] Wu M., Gharib M., Experimental studies on the shape and path of small air bubbles rising in clean water, Physics of Fluids 2002; Vol. 14, p. L 49

[19] de Vries J. et al., Induced bubble shape oscillations and their impact on the rise velocity, The European Physical Journal Bm 2002; Vol. 29, p. 503

[20] Zimmerman W.B., et al., Design of an airlift bioreactor and pilot scale studies with fluidic oscillator induced microbubbles for growth of a microalgae Dunaliella Salina, Applied Energy 2011; Vol. 88, p. 3357

[21] Zimmerman W.B., Tesař $\mathrm{V}$, Bubble generation for aeration and other purposes, filed Oct. 2006, British Patent GB20060021561 